\title{
Insecticide resistance modifies mosquito response to DEET and natural repellents
}

\author{
Emilie Deletre ${ }^{1,2^{*}} \mathbb{B}$, Thibaud Martin ${ }^{1,2}$, Claire Duménil ${ }^{1}$ and Fabrice Chandre ${ }^{3}$
}

\begin{abstract}
Background: Pyrethroid and organophosphate resistance in the malaria vector Anopheles gambiae has led to the search for not only alternative insecticides, but also repellent chemical compounds. However, little is known about the potential actions of repellents and the cross-resistance risk between insecticide and repellent compounds.

Methods: Here we show the action of permethrin, DEET, geraniol, carvacrol, culminaldehyde and cinnamaldehyde against three A. gambiae strains: 'Kis' (Kisumu susceptible strain), 'KdrKis' (pyrethroid resistant strain) and 'AcerKis' (organophosphate resistant strain), the last two differing from the first by a mutation on the $k d r$ and ace 1 genes, respectively.

Conclusions: Results from the DEET assays show it induced repellency for the resistant KdrKis and AcerKis strains but maintained irritancy for the susceptible strain. More generally, we show resistance genes modify the behavior of An. gambiae, increasing or decreasing the effectiveness of DEET and natural compounds, depending on the mutation. These findings offer a new avenue for research on the target and mechanism of repellent compounds. We discuss these findings in the context of vector control strategies.
\end{abstract}

Keywords: Anopheles gambiae, Permethrin, Deterrent, Mortality, Geraniol, Cinnamaldehyde

\section{Background}

The Anopheles gambiae (Giles, 1902) complex includes major vectors responsible for the transmission of Plasmodium spp., causing malaria infections in humans [1]. The enormous progress in rapid diagnostic tests (associated with efficient treatments such as artemisinin-based combination therapy against $P$. falciparum) and vector control with indoor residual spraying or long-lasting insecticide treated nets, has resulted in an overall decrease in malaria deaths [2]. Relatively safe for humans, pyrethroids have rapid irritant (or excito-repellent), knockdown and killing effects [3], mediated by modifying the gating kinetic of the voltage-dependent sodium channel. Pyrethroids of type I (e.g. permethrin) prevent the sodium channels from closing, creating a succession of repetitive action potentials, while the sodium channels in type II pyrethroids (e.g. deltamethrin) stay open without producing repetitive discharge [4]. Pyrethroids can be

\footnotetext{
${ }^{*}$ Correspondence: emilie.deletre@cirad.fr

${ }^{1}$ Cirad - Montpellier University - UPR Hortsys, Montpellier, France

Full list of author information is available at the end of the article
}

used for indoor spraying, and they are also used for treating bednets or cloths [5]. A common form of resistance to pyrethroids in An. gambiae is knockdown resistance $(k d r)$, resulting from a mutation (L1014F or L1014S) of the voltage-dependent sodium channel gene $\left(\mathrm{Na}_{\mathrm{v}}\right)[6,7]$. These mutations reduce the affinity of pyrethroids to $\mathrm{Na}_{\mathrm{v}}$ [8]. Organophosphates (OPs) are mainly used for indoor residual spraying, although only a few are recommended by the World Health Organization. Indeed, OPs do not have any irritant or repellent effect, they kill the mosquitoes after landing on the wall surfaces. OP insecticides inhibit acetylcholinesterase, a key enzyme of the nervous system [9]. Acetylcholinesterase (AChE) degrades the neurotransmitter at the cholinergic nerve synapse. When inhibited, acetylcholine accumulates in the synaptic junction and the receptors remain open, inducing paralysis and death [10-12]. For several mosquito species (including An. gambiae), a G119S substitution in AChE1 encoded by acwie-1 gene has been implicated in resistance to OP and carbamate insecticides [13-15]. The most common active ingredient in insect repellent (especially against mosquitoes), $N, N$-diethyl- $m$-toluamide (DEET), 
inhibits olfactory neuron receptors and masks attractive odours in An. gambiae [16, 17]. In vitro, DEET was also found to be an acetylcholinesterase inhibitor in mosquitoes [18], and Stanczyk et al. [19] also identified Aedes aegypti females that were insensitive to DEET, but no males, due to a genetically determined dominant trait and residing in changes in the sensillum function. Insecticide-based strategies have contributed to improving public health in many countries [20]. Nevertheless, vector control could be under the threat of continuous selection for resistant populations to insecticides. Indeed, pyrethroid and OP resistance have been reported in 27 countries from sub-Saharan Africa, with multiple resistance mechanisms, such as decreased sensitivity of the target protein and increased metabolic detoxification, underscoring the need to find alternatives to these chemical insecticides [2, 7, 9, 21-24]. Insecticide resistance can impact the behavioral response. Studies have previously shown that a $k d r$-resistant strain of $A$. gambiae is less affected by pyrethroids than the susceptible strain [25]. Although some studies showed that $k d r$ resistance failed to decrease the effectiveness of insecticide-treated nets [26], others reported a fitness advantage for $k d r$ resistant phenotypes [22] that could decrease the efficacy of pyrethroid treated nets [27].

Generally, the search for novel compounds for vector control has focused on their toxic effects. Nevertheless, research on other effects (such as repellency or irritancy) that may be used to reduce vector-host contact are currently being undertaken, although few studies have focused on their effect on insecticide-resistant strains. In previous studies, we showed that $(E)$-cinnamaldehyde, the major component of cinnamon bark (Cinnamomum zeylanicum) essential oil; carvacrol, one of the major compounds of thyme leaf (Thymus vulgaris) essential oil; geraniol, one of the major compounds of citronella leaf (Cymbopogon winterianus) essential oil; and cuminaldehyde, the major compound of cumin seed (Cuminum cyminum) essential oil; could have a repellent, irritant and/or toxic effect on an An. gambiae susceptible strain (Kisumu) [28, 29]. The repellent chemicals identified to-date in plants are: (i) alkaloids that can affect the acetylcholine receptors in the nervous system [30] or membrane channels of nerves [31]; (ii) phenols, particularly in the flavonoid group; and (iii) terpenoids, the most important insect repellent group to consider. For example, monoterpenes penetrate the insect cuticle, which increases their bioavailability [32]. This property could be of interest if it resulted in shortened stay of insects on treated surfaces. Three pathways have been studied to explain the toxicity of essential oils: (i) the inhibition of the acetylcholinesterase; (ii) interference with the neuromodulator octopamine; and (iii) inhibition of
GABA-gated chloride channels [33-35]. While the mode of action of repellency and irritancy has not been well studied, repellents could function through the activation (or inactivation) of olfactory receptor neurons and irritants through the activation of gustatory receptor neurons [36, 37].

The overall purpose of this article is to examine how insecticide resistance genes modify the behaviour of the mosquito Anopheles gambiae exposed to DEET and natural repellent compounds. Our specific aims are to identify the risk of cross-resistance between insecticides and repellents, and elicit more information about their potential mechanisms. In three behavioural assays, DEET and four bioactive repellent compounds were tested on a pyrethroid ( $k d r$ gene) and an OP (ace1 gene) resistant strain compared to a susceptible one, in comparison with permethrin and non-treated control. In this study, we tested: (i) spatial repellency (also called expellent repellency) which corresponds to oriented movement of the insect away from a volatile chemical source without direct contact; (ii) contact repellency (also called irritancy landing inhibition or excito-repellency) which corresponds to oriented movement of the insect away from a chemical source with direct contact; and (iii) contact toxicity [37].

\section{Results}

\section{DEET is a repellent to the $A$. gambiae resistant strain}

DEET failed to show any repellency on the susceptible Kis strain at low and high doses but showed significant repellency at high concentration for the pyrethroidresistant strains KdrKis (29.2\%) and OP-resistant strain AcerKis (85.7\%), compared with the non-treated control (Table 1, Additional file 1: Figure S1). Moreover, the repellent effect was significantly higher on the OPresistant strain AcerKis than on the susceptible strain Kis (15\%). DEET was an irritant at high concentration for all strains without significant difference between them (Table 2, Additional file 2: Figure S2). DEET was toxic at high concentration on the susceptible strain Kis (98.2\%) and the OP-resistant strain AcerKis strain (96.6\%), but not on the pyrethroid-resistant strain KdrKis (20.9\%) (Table 3, Additional file 3: Figure S3).

\section{Insecticide resistance modifies mosquito behavior to natural compounds}

Carvacrol showed a significant repellent effect at high concentration for the susceptible strain Kis and the pyrethroid-resistant KdrKis strain, but not on the OPresistant AcerKis strain (Table 1, Additional file 1: Figure S1). Carvacrol was an irritant at high concentration on the three strains but significantly less for both resistant strains KdrKis (45.3\%) and AcerKis (49.3\%), when 
Table 1 Repellent effect of DEET, permethrin, carvacrol, geraniol, cuminaldehyde and cinnamaldehyde on Anopheles gambiae from reference strains, the susceptible Kisumu strain (Kis), the pyrethroid-resistant strain (KdrKis) and the OP-resistant strain (AcerKis)

\begin{tabular}{|c|c|c|c|c|}
\hline Product & Concentration $\left(\mu \mathrm{l} / \mathrm{cm}^{2}\right)$ & Kis strain ${ }^{\mathrm{ab}}$ & KdrKis strain ${ }^{\text {ab }}$ & AcerKis strain ${ }^{\mathrm{ab}}$ \\
\hline \multirow[t]{2}{*}{ Permethrin } & 0.010 & $0.0(0.0-4.9)$ & $4.3(0.0-10.6)$ & $2.7(0.0-9.6)$ \\
\hline & 0.100 & $6.3(0.0-14.0)$ & $11.0(2.9-19.1)$ & $8.0(0.0-16.7)$ \\
\hline \multirow[t]{2}{*}{ DEET } & 0.010 & $0.0(0.0-1.4)$ & $20.1(9.2-31.0)$ & $11.9(2.6-21.2)$ \\
\hline & 0.100 & $15.0(5.2-24.7)$ & $29.2(17.4-40.9)$ & $85.7(77.6-93.8)^{*}$ \\
\hline \multirow[t]{2}{*}{ Carvacrol } & 0.001 & $9.4(1.7-17.0)$ & $11.2(3.1-19.3)$ & $5.7(0.0-13.5)$ \\
\hline & 0.014 & $36.6(25.0-48.2)$ & $42.0(30.1-53.8)$ & $0.0(0.0-3.6)^{*}$ \\
\hline \multirow[t]{2}{*}{ Geraniol } & 0.002 & $5.1(0.0-13.0)$ & $3.1(0.0-9.5)$ & $22.1(9.4-34.8)$ \\
\hline & 0.023 & $50.5(38.0-63.1)$ & $41.0(28.9-53.1)$ & $9.7(0.0-20.2)^{*}$ \\
\hline \multirow[t]{2}{*}{ Cuminaldehyde } & 0.003 & $1.6(0.0-4.7)$ & $24.7(12.6-36.8)^{*}$ & $8.5(0.6-16.5)$ \\
\hline & 0.030 & $25.4(14.3-36.5)$ & $52.9(41.0-64.9)^{*}$ & $47.0(35.2-58.8)$ \\
\hline \multirow[t]{2}{*}{ Cinnamaldehyde } & 0.008 & $16.5(7.8-25.3)$ & $36.2(24.0-48.4)$ & $37.7(24.9-50.5)$ \\
\hline & 0.079 & $43.0(32.1-54.0)$ & $82.1(74.2-90.0)^{*}$ & $53.1(40.9-65.3)$ \\
\hline
\end{tabular}

a Proportion of escaping mosquitoes as a percentage corrected with the negative control to compare strains by pairs (confidence interval)

b Pairwise comparison of proportion was done using Fisher's test with the Holm's sequential Bonferroni correction method. Values in bold lettering are significantly different from the non-treated control. Values followed by an asterisk $\left(^{*}\right)$ are significantly different from the Kis strain

Table 2 Irritant effect of DEET, permethrin, carvacrol, geraniol, cuminaldehyde and cinnamaldehyde on Anopheles gambiae from reference strains, the susceptible Kisumu strain (Kis), the pyrethroid-resistant strain KdrKis and the OP-resistant strain AcerKis

\begin{tabular}{|c|c|c|c|c|}
\hline Product & Concentration $\left(\mu \mathrm{l} / \mathrm{cm}^{2}\right)$ & Kis strain ${ }^{a b}$ & KdrKis strain ${ }^{\mathrm{ab}}$ & AcerKis strain ${ }^{\mathrm{ab}}$ \\
\hline \multirow[t]{2}{*}{ Permethrin } & 0.010 & $37.7(25.0-50.4)$ & $3.0(0.0-7.9)^{*}$ & $10.1(0.0-21.0) *$ \\
\hline & 0.100 & $25.9(13.8-38.0)$ & $45.6(33.7-57.4)$ & $27.9(16.7-39.1)$ \\
\hline \multirow[t]{2}{*}{ DEET } & 0.010 & $7.6(0.0-15.5)$ & $22.6(11.3-33.8)$ & $16.5(7.3-25.7)$ \\
\hline & 0.100 & $54.1(42.6-65.7)$ & $71.2(62.1-80.3)$ & $43.6(32.1-55.2)$ \\
\hline \multirow[t]{2}{*}{ Carvacrol } & 0.001 & $50.0(38.2-61.8)$ & $0.1(0.0-7.7)^{*}$ & $13.9(5.2-22.7)^{*}$ \\
\hline & 0.014 & $84.3(77.4-91.2)$ & $45.3(33.4-57.2)^{*}$ & $49.3(37.2-61.5)^{*}$ \\
\hline \multirow[t]{2}{*}{ Geraniol } & 0.002 & $16.5(5.2-27.7)$ & $24.8(14.5-35.2)$ & $19.6(9.1-30.2)$ \\
\hline & 0.023 & $45.9(41.4-50.4)$ & $73.9(65.7-82.0)^{*}$ & $41.8(30.4-53.3)$ \\
\hline \multirow[t]{2}{*}{ Cuminaldehyde } & 0.003 & $10.4(1.1-19.6)$ & $20.2(9.5-31.0)$ & $9.6(1.9-17.2)$ \\
\hline & 0.030 & $77.5(69.4-85.6)$ & $63.0(53.0-73.0)$ & $46.3(35.2-57.5)^{*}$ \\
\hline \multirow[t]{2}{*}{ Cinnamaldehyde } & 0.008 & $22.0(12.3-31.7)$ & $23.8(12.7-34.9)$ & $11.7(1.4-21.9)$ \\
\hline & 0.079 & $63.2(52.9-73.4)$ & $70.4(60.6-80.3)$ & $51.1(38.4-63.8)$ \\
\hline
\end{tabular}

a Proportion of escaping mosquitoes in percentage corrected with the negative control to compare strains by pairs (confidence interval)

b Pairwise comparison of proportion was done using Fisher's test with the Holm's sequential Bonferroni correction method. Values in bold lettering are significantly different from the non-treated control. Values followed by an asterisk $\left(^{*}\right)$ are significantly different from the Kis strain

compared to the susceptible strain Kis (84.3\%) (Table 2, Additional file 2: Figure S2). Actually, carvacrol was an irritant at low concentration on the susceptible strain Kis but not on both resistant strains. Carvacrol was not toxic for any strains at low and high doses (Table 3, Additional file 3: Figure S3).

Geraniol had a significant repellent effect at high concentration for the susceptible strain Kis and the pyrethroid-resistant KdrKis strain, but not at low concentrations (Table 1, Additional file 1: Figure S1). Surprisingly, it was the opposite for the OP-resistant AcerKis strain, as geraniol showed repellent activity at the lower dose but not at the higher dose. Geraniol showed a significant irritant effect at high concentration on the three strains (Table 2, Additional file 2: Figure S2). Moreover, geraniol showed significantly more irritant effect on the pyrethroid-resistant strain KisKdr (73.9\%) than on the susceptible strain Kis (45.9\%). Geraniol was not toxic for 
Table 3 Toxic effects of DEET, permethrin, carvacrol, geraniol, cuminaldehyde and cinnamaldehyde on Anopheles gambiae from reference strains, the susceptible Kisumu strain (Kis), the pyrethroid-resistant strain KdrKis and the OP-resistant strain AcerKis

\begin{tabular}{|c|c|c|c|c|}
\hline Product & Concentration $\left(\mu \mathrm{l} / \mathrm{cm}^{2}\right)$ & Kis strain ${ }^{\mathrm{ab}}$ & KdrKis strain ${ }^{a b}$ & AcerKis strain ${ }^{a b}$ \\
\hline \multirow{2}{*}{ Permethrin } & 0.010 & $93.8(89.7-97.8)$ & $2.7(0.0-9.6)^{*}$ & $66.1(53.7-78.5)^{*}$ \\
\hline & 0.100 & $96.8(96.8-96.8)$ & $63.2(49.8-76.5)^{*}$ & $100.0(100.0-100.0)$ \\
\hline \multirow[t]{2}{*}{ DEET } & 0.010 & $2.6(0.0-8.6)$ & $0.0(0.0-1.2)$ & $0.0(0.0-0.0)$ \\
\hline & 0.100 & $98.2(98.2-98.2)$ & $20.9(9.7-32.0)^{*}$ & $96.6(96.6-96.6)$ \\
\hline \multirow[t]{2}{*}{ Carvacrol } & 0.001 & $4.3(0.0-9.0)$ & $3.3(0.0-9.8)$ & $0.0(0.0-0.0)$ \\
\hline & 0.014 & $0.0(0.0-0.0)$ & $0.0(0.0-0.0)$ & $0.0(0.0-0.0)$ \\
\hline \multirow[t]{2}{*}{ Geraniol } & 0.002 & $0.0(0.0-3.6)$ & $1.7(0.0-4.9)$ & $0.0(0.0-4.0)$ \\
\hline & 0.023 & $0.0(0.0-3.5)$ & $3.1(0.0-7.3)$ & $1.2(0.0-9.3)$ \\
\hline \multirow[t]{2}{*}{ Cuminaldehyde } & 0.003 & $0.0(0.0-0.0)$ & $1.3(0.0-5.5)$ & $0.0(0.0-0.0)$ \\
\hline & 0.030 & $11.1(3.4-18.9)$ & $3.8(0.0-9.9)$ & $30.9(17.4-44.5)^{*}$ \\
\hline \multirow[t]{2}{*}{ Cinnamaldehyde } & 0.008 & $0.0(0.0-0.0)$ & $0.0(0.0-0.0)$ & $0.2(0.0-3.8)$ \\
\hline & 0.079 & $45.9(32.9-58.8)$ & $93.9(87.2-100.6)^{*}$ & $89.9(81.9-97.9)^{*}$ \\
\hline
\end{tabular}

a Proportion of dead mosquitoes in percentage corrected with the negative control to compare strains by pairs

b Pairwise comparison of proportion was done using Fisher's test with the Holm's sequential Bonferroni correction method. Values in bold lettering are significantly different from the non-treated control. Values followed by an asterisk $\left(^{*}\right)$ are significantly different from the Kis strain

any strains at both doses (Table 3, Additional file 3: Figure S3).

Cuminaldehyde showed significantly more repellent activity for all strains at high concentration (Table 1, Additional file 1: Figure S1). Whatever the concentration (low or high), cuminaldehyde was significantly more repellent on the pyrethroid-resistant strain KdrKis (24.7 and 52.9\%, respectively) than on the susceptible strain Kis (1.6 and 25.4\%, respectively). Cuminaldehyde exhibited an irritant effect at high concentration on the three strains (Table 2, Additional file 2: Figure S2) but was significantly less of an irritant on the OP-resistant strain AcerKis (46.3\%) than on the susceptible strain Kis (77.5\%). Cuminaldehyde was not toxic for any strains at both doses, compared with the non-treated control (Table 3, Additional file 3: Figure S3). However, at a higher dose, cuminaldehyde was significantly more toxic on the OP-resistant strain AcerKis (30.9\%) than on the susceptible strain Kis (11.1\%).

Cinnamaldehyde showed significantly more repellent activity for all strains at high concentration (Table 1, Additional file 1: Figure S1). The compound showed significantly more repellency on the pyrethroid-resistant strain KdrKis (82.1\%) than on the susceptible strain Kis $(43.0 \%)$. At low concentration, cinnamaldehyde was still repellent on both resistant strains KdrKis and AcerKis, but not on the susceptible Kis strain, when compared with the non-treated control. Cinnamaldehyde showed an irritant effect at high concentration on the three strains, but not at the lower dose (Table 2,
Additional file 2: Figure S2). At the higher dose, cinnamaldehyde was toxic for all the strains as compared with the non-treated control (Table 3, Additional file 3: Figure S3), but cinnamaldehyde was significantly more toxic on the pyrethroid-resistant strain KdrKis (93.9\%) and the OP-resistant strain AcerKis (89.9\%) than on the susceptible strain Kis (45.9\%).

\section{Permethrin is an irritant and toxicant rather than a repellent}

At either dose, permethrin (control) did not act as a repellent for any strain, whether susceptible or resistant, compared with the non-treated control (Table 1, Additional file 1: Figure S1). At low concentration, permethrin showed a significant irritant action on the susceptible Kis strain (37.7\%) but not on the pyrethroid-resistant strain KdrKis (3.0\%) and OP-resistant strain AcerKis (10.1\%). However, permethrin at a high concentration had an irritant effect for every strain compared with the non-treated control (Table 2, Additional file 2: Figure S2). As expected, permethrin at low concentration was significantly toxic to Kis and AcerKis but not to KdrKis compared with the non-treated control (Table 3, Additional file 3: Figure S3). At high concentration, permethrin was significantly toxic for all strains but significantly less for the pyrethroid-resistant strain KdrKis (63.2\%) than for the susceptible Kis strain (96.8\%) (Table 3, Additional file 3: Figure S3). 


\section{Discussion}

DEET elicited no spatial repellent action for the susceptible strain Kis. Our results show, for the first time, a spatial repellent effect of DEET for the pyrethroidresistant strain $\mathrm{KdrKis}$ and a significantly greater effect for the OP-resistant strain AcerKis. DEET was an irritant for the resistant strains, suggesting a lack of cross-resistance. We observed a reduction of toxicity on KdrKis, but DEET is generally not used for that purpose. Although the mode of action of DEET has not been fully elucidated, it is known that this chemical interacts with several targets from the nervous system. Moreover, repellents can be defined in two different ways: a compound that causes a movement away from the odor source (spatial repellent) (no host odor in the bioassay) but also by a compound that prevents the recognition/location of the host (masking repellent), DEET could be a repellent of the second category [37]. The different kind of repellent and their associated bioassays are discussed in more details in [37]. DEET is a famous repellent but to our knowledge there are no publications describing a method to test DEET without a host (human, guinea pig, etc.); it has only been tested with a host, e.g. in the arm assay, hence considering only the masking effect and not the spatial effect. Actually, DEET is also known to inhibit olfactory neuron receptors, masking attractive odours in An. gambiae [16, 17]. However, a recent study showed that DEET activates the Ir $40 a^{+}$neurons in Drosophila melanogaster and when Ir 40 a receptors are silenced, flies lose the ability to avoid DEET [38]. Within the central nervous system DEET targets octopaminergic synapses and affects muscarinic receptors $[39,40]$. DEET was identified as an acetylcholinesterase inhibitor when tested on neurons in vitro [18] but from our study, there was no evidence that the repellent effect of DEET interferes with acetylcholinesterase activity in vivo. Our results suggest that acetylcholinesterase mutation (G119S) could enhance the DEET activity and increase its repellency against AcerKis. The similar high toxicity of DEET observed on AcerKis and Kis also suggests either that the steric effect of G119S mutation on acetylcholinesterase does not interfere with DEET affinity or that the primary target for DEET toxicity is not acetylcholinesterase, but other receptors from the central nervous system (e.g. octopamine receptors) [39]. Surprisingly, a significant reduction of DEET toxicity was observed against KdrKis sharing the same genetic background with Kis but being homozygous for L1014F mutation in the $\mathrm{Na}_{\mathrm{v}}$ channels. This suggests that DEET has more than a single mode of action and that complex interactions between different targets from the nervous system are involved in its toxic effect and would require further neurophysiologic investigations.
Our results show that insecticide resistance alleles (such as $k d r$ and ace1) could have a positive or negative impact on the effectiveness of carvacrol, geraniol, cuminaldehyde and cinnamaldehyde on the mosquito Anopheles gambiae, by modifying its behaviour (Table 4). We investigated the effects of these four natural bioactive compounds from essential oils, because of their promising effects on Kis, the susceptible strain of An. gambiae [29]. We observed a potentiation of the following effects on the pyrethroid-resistant strain KdrKis, compared with the susceptible strain: (i) an increase of the repellent effect of cuminaldehyde and cinnamaldehyde; (ii) an increase of the geraniol irritant effect, but a reduction of carvacrol effect; and (iii) an increase of cinnamaldehyde toxicity. On the OP-resistant strain AcerKis, we observed a reduction of the following compound effects: (i) a decrease of repellent effect for carvacrol, geraniol and cinnamaldehyde; (ii) a decrease of carvacrol and cuminaldehyde irritancy; and (iii) an increase of cinnamaldehyde toxicity. Comparison between the four bioactive compounds on a susceptible strain and two resistant strains showed differential effects that could be associated with the resistance mechanisms involved. Nowadays, few studies are conducted on the effect of natural compounds on the nervous system of insects. Most studies have investigated the effects of geraniol or carvacrol on AChE. Lopez \& Pascual-Villalobos [41] showed that geraniol was a weak reversible competitive inhibitor of AChE, although it has a strong insecticidal property on Sitophilus oryzae, Rhyzopertha dominica and Cryptolestes pusillus. Other studies deal with the inhibition of TRP channels that are involved in neurophysiological processes (such as photoreception, pheromone sensing, gustative perception, thermosensation, pain perception and mechanosensation [42]). Cinnamaldehyde and

Table 4 Summary of repellent, irritant and toxic effects of permethrin, DEET, carvacrol, geraniol, cuminaldehyde and cinnamaldehyde on Anopheles gambiae from reference strains, the susceptible Kisumu strain (Kis), the pyrethroid-resistant strain KdrKis and the OP-resistant strain AcerKis

\begin{tabular}{llll}
\hline Product & $\begin{array}{l}\text { Susceptible } \\
\text { strain } \\
\text { Kis }\end{array}$ & $\begin{array}{l}\text { Pyrethroid-resistant } \\
\text { strain } \\
k d r K i s\end{array}$ & $\begin{array}{l}\text { OP-resistant } \\
\text { strain } \\
\text { acerkis }\end{array}$ \\
\hline Permethrin & $\mathrm{R}^{0} \mathrm{I}^{+} \mathrm{T}^{++}$ & $\downarrow \mathrm{R}^{0} \mathrm{I}^{-} \mathrm{T}^{-}$ & $\downarrow \mathrm{R}^{0} \mathrm{I}^{-} \mathrm{T}^{+}$ \\
DEET & $\mathrm{R}^{0} \mathrm{I}^{+} \mathrm{T}^{+}$ & $\uparrow \mathrm{R}^{+} \mathrm{I}^{+} \mathrm{T}^{0}$ & $\uparrow \mathrm{R}^{++} \mathrm{I}^{+} \mathrm{T}^{+}$ \\
Carvacrol & $\mathrm{R}^{+} \mathrm{I}^{++} \mathrm{T}^{0}$ & $\downarrow \mathrm{R}^{+} \mathrm{I}^{-} \mathrm{T}^{0}$ & $\downarrow \mathrm{R}^{0} \mathrm{I}^{-} \mathrm{T}^{0}$ \\
Geraniol & $\mathrm{R}^{+} \mathrm{I}^{+} \mathrm{T}^{0}$ & $\uparrow \mathrm{R}^{+} \mathrm{I}^{++} \mathrm{T}^{0}$ & $\downarrow \mathrm{R}^{-} \mathrm{I}^{+} \mathrm{T}^{0}$ \\
Cuminaldehyde & $\mathrm{R}^{+} \mathrm{I}^{++} \mathrm{T}^{0}$ & $\uparrow \mathrm{R}^{++} \mathrm{I}^{++} \mathrm{T}^{0}$ & $\downarrow \mathrm{R}^{+} \mathrm{I}^{+} \mathrm{T}^{0}$ \\
Cinnamaldehyde & $\mathrm{R}^{+} \mathrm{I}^{+} \mathrm{T}^{+}$ & $\uparrow \mathrm{R}^{++} \mathrm{I}^{+} \mathrm{T}^{++}$ & $\uparrow \mathrm{R}^{+} \mathrm{I}^{+} \mathrm{T}^{++}$ \\
\hline
\end{tabular}

Abbreviations: $\mathrm{R}$, repellent; I, irritant; $\mathrm{T}$, toxic; 0 , no effect; + , effect; ++ , higher effect; - , lower effect; $\downarrow$, reduced efficiency for the strain; $\uparrow$, greater efficiency for the strain compared with the susceptible strain Kis 
carvacrol inhibit some TRP channels in Drosophila sp. $[43,44]$, suggesting that these natural compounds could act on several targets in the nervous central system. Geraniol had a lower repellent effect on acerKis, suggesting a possible interaction with the mutation of AChE. Cuminaldehyde, which is also an inhibitor of AChE [45], had a lower irritant effect and a higher toxic effect on AcerKis. The reduction in irritant effect tends to increase the contact of mosquitoes with the treated surfaces, and could contribute to increased mortality. In our study, carvacrol repellency and irritancy were weaker for the resistant AcerKis than the susceptible Kis. In vitro, carvacrol caused a slight inhibition of acetylcholinesterase from electric eels [46], and some arthropods (such as houseflies, ticks and cockroaches), but not from the mosquito Aedes aegypti [47]. The repellent effect of cinnamaldehyde and cuminaldehyde was higher on the strain with $k d r$ mutation than on the two others. These two compounds induced a strong response using an electroantennogram (EAG), suggesting a possible interaction with specific odor receptors and/or $\mathrm{Na}_{\mathrm{v}}$ channels [29]. In a previous study, we showed that geraniol elicited a significant response compared to ethanol using EAG, thus differing from carvacrol [29]. Since geraniol was also more of an irritant for KdrKis than for the other two strains, the mutated $\mathrm{Na}_{\mathrm{v}}$ channel could potentiate the interaction with geraniol, and afterward its irritancy. Conversely, the carvacrol irritant effect was lower for the KdrKis resistant strain. Because these compounds act differently on susceptible and resistant strains, this suggests that Cvp$\mathrm{dNa}$ and $\mathrm{AChE}$ could be primary or secondary targets of these compounds. Target site mutations could modify the sensitivity to these compounds, i.e. pleiotropic effects associated with resistance mechanisms that modify the behavioral response of resistant insects compared to susceptible ones. More studies on the affinity of natural compounds with the different receptors of the nervous system could enable researchers to identify new targets for repellent compounds or facilitate the discovery of new active molecules. The mode of action of these compounds should be further studied to determine how they first enter the insect, whether through ingestion, the respiratory route or cuticle absorption; for instance, volatile and/or topical routes of products can cause toxicity [48]. Some essential terpenes are also competitive inhibitors of acetylcholinesterase in vitro, but that may not correlate with toxicity, as evidenced by the case of carvacrol [33].

Our results confirm that permethrin is irritant and toxic but does not have a repellent effect for Anopheles gambiae $[28,29]$ as it does for other mosquito species (such as An. albimanus [49] or Aedes aegypti [50]). Indeed, permethrin is unlikely to volatilize based on its low vapor pressure $\left(6.9 \times 10^{-6} \mathrm{~Pa}\right.$ at $\left.25^{\circ} \mathrm{C}\right)$ and low
Henry's law constant [51]. Our results show permethrin at the lowest dose is less irritant and toxic for the pyrethroid-resistant strain KdrKis than for the susceptible strain Kis. This was also observed for the OP-resistant AcerKis strain, but at a lower intensity than for KdrKis, taking into account the knocked-down individuals. At a higher dose, permethrin was less of an irritant for the Kis strain, because the knockdown effect of some mosquitoes ( $\sim 30 \%$ individuals) during exposure prevented them from escaping, whereas permethrin became an irritant for the resistant KdrKis and AcerKis strains that failed to suffer knockdown. No knockdown effect was observed for the repellents. The knockdown effect is an early response of insects to an insecticide, leading to incapacitation, and occasionally, metabolic recovery [52]. Our results on the irritant effect of permethrin were influenced by its high and rapid toxicity, i.e. Kis females could not escape the cylinders, since a significant proportion were knocked down, whereas KdrKis were not knocked down and could escape. As previously reported by Chandre et al. [25], we confirmed that $A$. gambiae having $k d r$ mutation is resistant to the toxic and irritant effects of pyrethroid compared to the susceptible strain. Our results show, to a lesser extent, quite similar effects on the OP-resistant strain AcerKis with the lowest dose of permethrin. While the reduction of toxic and irritant effects on the KdrKisresistant strain threatens the effectiveness of insecticide nets treated with pyrethroids, our results and observations from the field suggest that when the dose is high enough, the reduced toxic effect is counter-balanced by the reduced irritancy, which increases the contact of mosquitoes with treated nets, leading to significant mortality of $k d r$-resistant mosquitoes. Hence, we showed that for type I pyrethroids (e.g. permethrin) the behavioural response of the KdrKis-resistant mosquito strain is modified, thus we can hypothesize that the behavioural response of the KdrKis-resistant mosquito strain will also be different toward type II pyrethroids (e.g. deltamethrin). Actually these pyrethroids have the same target, the sodium channels [4].

\section{Conclusions}

We show for the first time a repellent effect of DEET on the pyrethroid-resistant strain KdrKis of the mosquito Anopheles gambiae, and more significantly for the OPresistant strain AcerKis, compared to the susceptible strain Kis. Insecticide resistance genes (such as $k d r$ and ace1) could have a positive or negative impact on the effectiveness of natural repellent compounds such as carvacrol, geraniol, cuminaldehyde and cinnamaldehyde on An. gambiae, by modifying its behaviour. Although the mechanisms underlying the role of target site mutations on the response to irritant or repellent compounds 
are not yet known, it has already been described in the case of pyrethroid resistance. Wagman et al. [53] showed that Aedes aegypti with a decreased insecticide susceptibility were insensitive to the repellent effect of transfluthrin. In the same way, pyrethroid-resistant strains of An. gambiae or Culex quinquefasciatus were less irritated by permethrin compared to susceptible ones [22, 25-54]. This study underlines the importance of testing new compounds on strains with known resistance mechanisms, even if they do not seem to have strong effects on the strains, since the behavioural response of insects to repellent or irritant compounds can be higher or lower, depending on their target and mode of action. Our evidence highlights the need for improved knowledge on the modes of action of repellent/irritant products that could be promising alternatives or complementary tools, to overcome insecticide resistance in mosquito populations.

\section{Methods Insects}

Behavioural assays were performed using females of three reference strains of An. gambiae. The susceptible reference strain Kis (Kisumu susceptible strain) originally collected in Kisumu, Kenya in 1953, has been reared at LIN-IRD, Montpellier, France for more than 15 years. The insecticide susceptibility of the Kis strain was confirmed with World Health Organization (WHO) diagnostic doses (i.e. $4 \% \mathrm{DDT}, 0.75 \%$ permethrin) and is regularly controlled every 4 months as recommended by ISO 9001 . The colony was maintained in a climate-controlled room at $27 \pm 2{ }^{\circ} \mathrm{C}, 80 \pm 10 \% \mathrm{RH}$, with a photoperiod cycle of $12 \mathrm{~h}$ light: $12 \mathrm{~h}$ dark. Mosquito larvae were fed a diet of fish food (TetraMin, Tetra, Montpellier, France). Emerged adults were mechanically aspirated and transferred into $25 \times 25 \times 25 \mathrm{~cm}$ cages and provided access to $10 \%$ honeywater solution. The biological assays were also performed on females of An. gambiae from two resistant strains: the pyrethroid-resistant strain KdrKis (which is homozygous for the $k d r$ L1014F mutation with the same genetic background as the Kis strain) and the organophosphate/ carbamate-resistant strain AcerKis (which is homozygous for G119S ace-1 mutation and has the same genetic background as the susceptible Kis strain) $[5,6]$. The susceptible and resistant populations were reared in separate insectaries.

\section{Products}

Studies were performed with four natural products: $(E)$ cinnamaldehyde ( $99 \%$ purity), geraniol ( $98 \%$ purity), cuminaldehyde (98\% purity) and carvacrol ( $\geq 98 \%$ purity) (Sigma-Aldrich, St Louis, MO, USA). Two synthetic products were also tested: $N, N$-diethyl-3-methylbenzamide (also known as diethyltoluamide) (DEET, 97\% purity) and permethrin (99\% purity) from Sigma-Aldrich. The pyrethroid permethrin (mainly used in mosquito nets) and the insect repellent DEET (which is effective at reducing mosquito bites [55-57]), were used as positive controls. DEET and permethrin were diluted at 0.1 and $1 \%(\mathrm{v} / \mathrm{v})$ in a solvent that consisted of ethanol $(2 / 3)$ and Dow Corning ${ }^{\circledR} 556$ cosmetic grade fluid (1/3). In a previous study we proved the four natural compounds (cinnamaldehyde, culminaldehyde, geraniol and carvacrol) were repellent, irritant and/or toxic at the relative concentration of $0.1 \%$ found in their respective essential oils (cinnamon, cumin, lemongrass and thyme essential oils [28, 29]). These products were tested at 2 concentrations: this efficient concentration, and diluted 10 times. These dilutions ensured that the quantity of a compound tested was approximately the same within the essential oil. Cinnamaldehyde was tested at $0.008 \mu \mathrm{l} / \mathrm{cm}^{2}$ of chromatograph paper and $0.079 \mu \mathrm{l} / \mathrm{cm}^{2}$, cuminaldehyde at 0.003 and $0.030 \mu \mathrm{l} / \mathrm{cm}^{2}$, geraniol at 0.002 and $0.023 \mu \mathrm{l} / \mathrm{cm}^{2}$, and carvacrol at 0.001 and $0.014 \mu \mathrm{l} / \mathrm{cm}^{2}$. Evaluation of a negative control with the solvent ethanol-silicone fluid preceded each assay (Table 2, Additional file 2: Figure S2). In repellency assays, $3.3 \mathrm{ml}$ of this solution was deposited on a $13 \times 30 \mathrm{~cm}$ chromatography paper, except on a border margin of $1.5 \mathrm{~cm}$ width. For irritancy and toxicity assays, $2 \mathrm{ml}$ of the solution was deposited on $12 \times 15 \mathrm{~cm}$ chromatography paper.

\section{Behavioral bioassays}

Detailed descriptions of the apparatus, assay protocols, and data analysis procedures have already been published [28], modified from Grieco et al. [58]. In summary, bioassays were conducted between 10:00 and 18:00 h at $24 \pm 1{ }^{\circ} \mathrm{C}$ and $50 \pm 10 \% \mathrm{RH}$, and for each product; all assays were performed the same day.

\section{Repellency assays}

The apparatus was a cylinder divided into two chambers, one treated and the other untreated. Treated papers (with products or with only the solvent, as control) were rolled around to cover the inner surface of the treated chamber, whereas untreated chromatograph paper was used to cover the inner surface of the untreated chamber. A metallic grid prevented direct mosquito contact with the treated paper. Twenty non-blood-fed females (aged 4-7 days-old) were introduced in the treated chamber and after a $30 \mathrm{~s}$ acclimation period, the butterfly valve separating the two chambers was opened for $10 \mathrm{~min}$. At the end of the test, the butterfly valve was closed and the number of insects in each chamber recorded. Mosquitoes moving from the treated chamber to the untreated chamber were recorded as 'escaped. Conversely, mosquitoes remaining in the treated chamber were recorded 
as 'stayed. Tests were replicated three times for each chemical.

\section{Irritancy assays}

These assays were performed using the system described for the repellent assay, and consisted of two connected tubes used in the WHO test kit, and a possible mosquito contact with the chemical. Ten non-blood-fed females (aged 4-7 days-old) were introduced in the treated chamber and each test performed six times for each chemical. After a $30 \mathrm{~s}$ acclimation period, the guillotine valve separating the two chambers was opened for $10 \mathrm{~min}$, allowing the mosquitoes to move freely throughout the arena. Once the guillotine valve was closed, the number of mosquitoes in each tube ('stayed' $v s$ 'escaped') was recorded.

\section{Toxicity assays}

Toxicity assays were performed using a WHO Test Kit [59]. Twenty non-blood-fed females (aged 4-7 days-old) were exposed for $1 \mathrm{~h}$ to a treated paper (with products or with the solvent only) in the treated tube. Mosquitoes were then transferred to an untreated tube with $10 \%$ honey solution and maintained at $27{ }^{\circ} \mathrm{C}$ and $80 \% \mathrm{RH}$. The number of dead and alive An. gambiae was recorded after $24 \mathrm{~h}$. Each test was replicated three times for each chemical.

\section{Statistical analysis}

The same method was used to analyse the proportion of dead mosquitoes in toxicity assays and the proportion of escaped mosquitoes in both repellency and irritancy assays. Data analysis was carried out using $\mathrm{R}$ software v.2.12.2. Tests of treatment effects for the different behavioural assays were carried out on the proportion of escaped or dead mosquitoes in (i) control and treated assays; and (ii) susceptible and resistant strain assays. Fisher's exact test with Bonferroni correction using Holm's sequential method [60] was used for repellency and irritancy. The behavioural and mortality data were corrected using Sun-Shepard's formula before comparing the susceptible strain with the resistant ones [61].

\section{Additional files}

Additional file 1: Figure S1. Repellent effect of DEET, permethrin, carvacrol, geraniol, cuminaldehyde and cinnamaldehyde on Anopheles gambiae from the susceptible Kisumu strain (Kis), the pyrethroid resistant strain KdrKis and the OP resistant strain AcerKis.

Additional file 2: Figure S2. Irritancy effect of DEET, permethrin, carvacrol, geraniol, cuminaldehyde and cinnamaldehyde on Anopheles gambiae from the susceptible Kisumu strain (Kis), the pyrethroid resistant strain KdrKis and the OP resistant strain AcerKis.
Additional file 3: Figure S3. Toxicity effect of DEET, permethrin, carvacrol, geraniol, cuminaldehyde and cinnamaldehyde on Anopheles gambiae from the susceptible Kisumu strain (Kis), the pyrethroid resistant strain KdrKis and the OP resistant strain AcerKis.

\section{Abbreviations}

$\mathrm{Na}_{\mathrm{v}}$ : voltage-dependent sodium channel gene; Kis: Kisumu susceptible strain; KdrKis: pyrethroid resistant strain; AcerKis: organophosphate resistant strain; OPs: organophosphates; AChE: acetylcholinesterase; WHO: World Health Organisation; DEET: N,N-diethyl-3-methylbenzamide; RH: relative humidity; EAG: electroantennogram.

\section{Acknowledgments}

We thank M. N. Lacroix and C. Ginibre for rearing and providing the mosquitoes. We thank Cirad and IRD, Montpellier, France and also Mutavie, Paris, France, for supporting this work. ED and TM also gratefully acknowledge financial support for this research from icipe core funding provided by UK Aid from the UK Government, Swedish International Development Cooperation Agency (Sida), the Swiss Agency for Development and Cooperation (SDC), Federal Ministry for Economic Cooperation and Development (BMZ), Germany, and the Kenyan Government. The views expressed herein do not necessarily reflect the official opinion of the donors.

\section{Funding}

This study was supported by the foundation Mutavie, Paris, France.

\section{Availability of data and materials}

Data supporting the conclusions of this article are included within the article. The datasets used and/or analysed during the present study are available from the corresponding author upon reasonable request.

\section{Authors' contributions}

Study concept and design: ED and FC. Biological study: CD and ED. Analysis and interpretation of data: $C D$ and ED. Drafting of the manuscript: ED. Material support: ED and FC. Critical revision of the manuscript: TM and FC. All authors read and approved the final manuscript.

Ethics approval and consent to participate Not applicable.

\section{Consent for publication}

Not applicable.

\section{Competing interests}

The authors declare that they have no competing interests.

\section{Publisher's Note}

Springer Nature remains neutral with regard to jurisdictional claims in published maps and institutional affiliations.

\section{Author details}

${ }^{1}$ Cirad - Montpellier University - UPR Hortsys, Montpellier, France. ${ }^{2}$ International Centre of Insect Physiology and Ecology (icipe), Nairobi, Kenya. ${ }^{3}$ UMR MIVEGEC, IRD-CNRS-Montpellier University, Montpellier, France.

Received: 28 August 2018 Accepted: 26 February 2019

Published online: 12 March 2019

\section{References}

1. WHO. Mise en oeuvre de la stratégie mondiale de lutte antipaludique. Technical Report Series 839. Geneva: World Health Organization; 1993.

2. WHO. World health statistics 2011. WHO Statistical Information System (WHOSIS). Geneva: World Health Organization; 2011. http://www.who. int/whosis/whostat/2011/en/. Accessed 1 Mar 2017.

3. Zaim M, Aitio A, Nakashima N. Safety of pyrethroid-treated mosquito nets. Med Vet Entomol. 2000;14:1-5. 
4. Lund $A E$, Narahashi T. Kinetics of sodium channel modification as the basis for the variation in the nerve membrane effects of pyrethroids and DDT analogs. Pest Biochem Physiol. 1983;20:203-16.

5. WHO. WHO recommended long-lasting insecticidal mosquito nets. Geneva: World Health Organization; 2009. http://www.who.int/whopes/ Long_lasting_insecticidal_nets_Jul_2012.pdf. Accessed 13 July 2012.

6. Martinez-Torres D, Chandre F, Williamson MS, Darriet F, Berge JB, Devonshire AL, et al. Molecular characterization of pyrethroid knockdown resistance $(\mathrm{kdr})$ in the major malaria vector Anopheles gambiae s.s. Insect Mol Biol. 1998;7:179-84

7. Ranson H, Jensen B, Vulule JM, Wang X, Hemingway J, Collins FH. Identification of a point mutation in the voltage-gated sodium channel gene of Kenyan Anopheles gambiae associated with resistance to DDT and pyrethroids. Insect Mol Biol. 2000;9:491-7.

8. Pauron D, Barhanin J, Amichot M, Pralavorio M, Berge JB, Lazdunski M. Pyrethroid receptor in the insect $\mathrm{Na}+$ channel: alteration of its properties in pyrethroid-resistant flies. Biochem J. 1989;28:1673-7.

9. Liu N. Insecticide resistance in mosquitoes: Impact, mechanisms, and research directions. Annu Rev Entomol. 2015;60:537-59.

10. Aldridge WN. Some properties of specific cholinesterase with particular reference to the mechanism of inhibition by diethyl p-nitrophenyl thiophosphate (E 605) and analogues. Biochem J. 1950;46:451-60.

11. Fukuto TR. Mechanism of action of organophosphorus and carbamate insecticides. Environ Health Perspect. 1990;87:245-54.

12. Fournier D, Mutero A. Modification of acetylcholinesterase as a mechanism of resistance to insecticides. Comp Biochem Physiol C Comp Pharmacol Toxicol. 1994;108:19-31.

13. Weill M, Fort P, Berthomieu A, Dubois MP, Pasteur N, Raymond M. A novel acetylcholinesterase gene in mosquitoes codes for the insecticide target and is non-homologous to the ace gene in Drosophila. Proc Biol Sci. 2002:269:2007-16.

14. Weill M, Lutfalla G, Mogensen K, Chandre F, Berthomieu A, Berticat C, et al. Comparative genomics: insecticide resistance in mosquito vectors. Nature. 2003;423:136-7.

15. Alout H, Djogbénou L, Berticat C, Chandre F, Weill M. Comparison of Anopheles gambiae and Culex pipiens acetylcholinesterase 1 biochemical properties. Comp Biochem Physiol B Biochem Mol Biol. 2008;150:271-7.

16. Ditzen $M$, Pellegrino $M$, Vosshall LB. Insect odorant receptors are molecular targets of the insect repellent DEET. Science. 2008;319:1838-42.

17. DeGennaro M, McBride CS, Seeholzer L, Nakagawa T, Dennis EJ, Goldman $C$, et al. orco mutant mosquitoes lose strong preference for humans and are not repelled by volatile DEET. Nature. 2013;498:487-91.

18. Corbel V, Stankiewicz M, Pennetier C, Fournier D, Stojan J, Girard E, et al. Evidence for inhibition of cholinesterases in insect and mammalian nervous systems by the insect repellent DEET. BMC Biol. 2009;7:47.

19. Stanczyk NM, Brookfield JFY, Ignell R, Logan JG, Field LM. Behavioral insensitivity to DEET in Aedes aegypti is a genetically determined trait residing in changes in sensillum function. Proc Natl Acad Sci USA. 2010;107:8575-80.

20. WHO. World Health Statistics 2008. WHO Statistical Information System (WHOSIS), Geneva: World Health Organization; 2008. http://www.who. int/whosis/whostat/2008/en/. Accessed 20 May 2008.

21. Akogbeto M, Yakoubou S. Resistance of malaria vectors to pyrethroids used for impregnated bednets, Benin, West Africa. Bull Soc Path Exot. 1999:92:123-30

22. Ranson H, N'Guessan R, Lines J, Moiroux N, Nkuni Z, Corbel V. Pyrethroid resistance in African anopheline mosquitoes: what are the implications for malaria control? Trends Parasitol. 2011:27:91-8.

23. Corbel V, Chandre F, Brengues C, Akogbéto M, Lardeux F, Hougard JM, Guillet P. Dosage-dependent effects of permethrin-treated nets on the behaviour of Anopheles gambiae and the selection of pyrethroid resistance. Malar J. 2004;8:22.

24. WHO. Global plan for insecticide resistance management in malaria vectors. Geneva: World Health Organization; 2012

25. Chandre F, Darriet F, Duchon S, Finot L, Manguin S, Carnevale P, et al. Modifications of pyrethroid effects associated with kdr mutation in Anopheles gambiae. Med Vet Entomol. 2000;14:81-8.

26. Henry MC, Assi SB, Rogier C, Dossou-Yovo J, Chandre F, Guillet P, et al. Protective efficacy of lambda-cyhalothrin treated nets in Anopheles gambiae pyrethroid resistance areas of Côte d'Ivoire. Am J Trop Med Hyg. 2005:73:859-64
27. N'Guessan R, Corbel V, Akogbéto M, Rowland M. Reduced efficacy of insecticide-treated nets and indoor residual spraying for malaria control in pyrethroid resistance area, Benin. Emerg Infect Dis. 2007;13:199-206.

28. Deletre E, Martin T, Campagne P, Bourguet D, Cadin A, Menut C, et al. Repellent, irritant and toxic effects of 20 plant extracts on adults of the malaria vector Anopheles gambiae mosquito. PLoS One. 2013;8:e82103.

29. Deletre $E$, Chandre F, Williams L, Duménil C, Menut C, Martin T. Electrophysiological and behavioral characterization of bioactive compounds of the Thymus vulgaris, Cymbopogon winterianus, Cuminum cyminum and Cinnamomum zeylanicum essential oils against Anopheles gambiae and prospects for their use as bednet treatments. Parasit Vectors. 2015;8:316.

30. Flattum RF, Shankland DL. Acetylcholine receptors and the diphasic action of nicotine in the American cockroach, Periplaneta americana (L.). Comp General Pharmacol. 1971;2:159-67.

31. Amar M, Pichon Y, Inoue I. Micromolar concentrations of vetradine activate sodium channels in embryonic cockroach neurons in culture Pflugers Arch. 1991;417:500-8.

32. Moretti AN, Zerba EN, Alzogaray RA. Behavioral and toxicological responses of Rhodnius prolixus and Triatoma infestans (Hemiptera: Redu viidae) to 10 monoterpene alcohols. J Med Entomol. 2013;50:1046-54.

33. Isman MB. Plant essential oils for pest and disease management. Crop Prot. 2000;19:603-8.

34. Isman MB. Botanical insecticides, deterrents, and repellents in modern agriculture and an increasingly regulated world. Annu Rev Entomol. 2006;51:45-66.

35. Regnault-Roger C, Vincent C, Arnason JT. Essential oils in insect control: low-risk products in a high-stakes world. Annu Rev Entomol. 2011;57:405-24.

36. Dekker T, Ignell R, Ghebru M, Glinwood R, Hopkins R. Identification of mosquito repellent odours from Ocimum forskolei. Parasit Vectors. 2011:4:183.

37. Deletre E, Schatz B, Bourguet D, Chandre F, Williams L, Ratnadass A, et al. Prospects for repellent in pest control: current developments and future challenges. Chemoecology. 2016;26:127-42.

38. Kain P, Boyle SM, Tharadra SK, Guda T, Pham C, Dahanukar A, et al. Odour receptors and neurons for DEET and new insect repellents. Nature. 2013;502:507-12.

39. Swale DR, Sun B, Tong F, Bloomquist JR. Neurotoxicity and mode of action of N,N-diethyl-meta-toluamide (DEET). PLoS One. 2014;9:e103713.

40. Abd-Ella A, Stankiewicz M, Mikulska K, Nowak W, Pennetier C, Goulu $M$, et al. The repellent DEET potentiates carbamate effects via insect muscarinic receptor interactions: an alternative strategy to control insect vector-borne diseases. PLoS One. 2015;10:e0126406.

41. López MD, Pascual-Villalobos MJ. Mode of inhibition of acetylcholinesterase by monoterpenoids and implications for pest control. Ind Crops Prod. 2010;31:284-8

42. Moran MM, Xu H, Clapham DE. TRP ion channels in the nervous system. Curr Opin Neurobiol. 2004;14:362-9.

43. Nagata K. TRP channels as target sites for insecticides: physiology, pharmacology and toxicology. Invert Neurosci. 2007;7:31-7.

44. Parnas M, Peters M, Dadon D, Lev S, Vertkin I, Slutsky I, et al. Carvacrol is a novel inhibitor of Drosophila TRPL and mammalian TRPM7 channels. Cell. $2009 \cdot 45 \cdot 300-9$

45. Abdelgaleil SA, Mohamed MI, Badawy ME, El-arami SA. Fumigant and contact toxicities of monoterpenes to Sitophilus oryzae (L.) and Tribolium castaneum (Herbst) and their inhibitory effects on acetylcholinesterase activity. J Chem Ecol. 2009;35:518-25.

46. Jukic M, Politeo O, Maksimovic M, Milos M, Milos M. In vitro acetylcholinesterase inhibitory properties of thymol, carvacrol and their derivatives thymoquinone and thymohydroquinone. Phytother Res. 2007;21:259-61.

47. Anderson JA, Coats JR. Acetylcholinesterase inhibition by nootkatone and carvacrol in arthropods. Pestic Biochem Physiol. 2012;102:124-8.

48. Regnault-Roger C, Hamraoui A. Lutte contre les insectes phytophages par les plantes aromatiques et leurs molécules allélochimiques. Acta Bot Gallica. 1997;144:401-12.

49. Dusfour I, Achee NL, Roberts DR, Grieco JP. Contact irritancy and repellency behaviors in Anopheles albimanus Wiedemann (Diptera: Culicidae) collected in Orange Walk, Belize, C.A. J Vector Ecol. 2009;34:232-7.

50. Achee NL, Sardelis MR, Dusfour I, Chauhan KR, Grieco JP. Characterization of repellent, contact irritant, and toxicant chemical actions of standard vector control compounds. J Am Mosq Control Assoc. 2009;25:156-67. 
51. Imgrund $\mathrm{H}$. Environmental fate of permethrin. Sacramento, CA, USA: Department of Pesticide Regulation; 2003.

52. White G. Terminology of insect repellents. In: Debboun M, Frances SP, Strickman D, editors. Insect repellents: principles, methods and uses. Boca Raton: CRC Press; 2007.

53. Wagman JM, Achee NL, Grieco JP. Insensitivity to the spatial repellent action of transfluthrin in Aedes aegypti: a heritable trait associated with decreased insecticide susceptibility. PLoS NegI Trop Dis. 2015;9:e0003726.

54. Boonyuan W, Bangs MJ, Grieco JP, Tiawsirisup S, Prabaripai A, Chareonviriyaphap T. Excito-repellent responses between Culex quinquefasciatus permethrin susceptible and resistant mosquitoes. J Ins Behav. 2016;29:415-31.

55. Curtis CF, Lines JD, ljumba J, Callaghan A, Hill N, Karimzad MA. The relative efficacy of repellents against mosquito vectors of disease. Med Vet Entomol. 1987;1:109-19.

56. Badolo A, Ilboudo-Sanogo E, Ouédraogo AP, Costantini C. Evaluation of the sensitivity of Aedes aegypti and Anopheles gambiae complex mosquitoes to two insect repellents: DEET and KBR 3023. Trop Med Int Health. 2004;9:330-4.
57. Costantini C, Badolo A, Ilboudo-Sanogo E. Field evaluation of the efficacy and persistence of insect repellents DEET, IR3535, and KBR 3023 against Anopheles gambiae complex and other Afrotropical vector mosquitoes. Trans R Soc Trop Med Hyg. 2004;98:644-52.

58. Grieco JP, Achee NL, Sardelis MR, Chauhan KR, Roberts DR. A novel high-throughput screening system to evaluate the behavioural response of adult mosquitoes to chemicals. J Am Mosq Control Assoc. 2005;21:404-11.

59. WHO. Report of the WHO informal consultation. Test procedures for insecticide resistance monitoring in malaria vectors, bio-efficacy and persistence of insecticides on treated surfaces. WHO/CDS/CPC/MAL/98(12). Geneva: World Health Organization; 1998.

60. Holm S. A simple sequentially rejective multiple test procedure. Scand J Stat. 1979:6:65-70.

61. Püntener W. Manual for field trials in plant protection. 2nd ed. Basel: CibaGeigy Limited; 1981.
Ready to submit your research? Choose BMC and benefit from:

- fast, convenient online submission

- thorough peer review by experienced researchers in your field

- rapid publication on acceptance

- support for research data, including large and complex data types

- gold Open Access which fosters wider collaboration and increased citations

- maximum visibility for your research: over 100M website views per year

At BMC, research is always in progress.

Learn more biomedcentral.com/submissions 